\title{
Automatically extracting contours of cross-sections from 3D point clouds of tunnel
}

\author{
Wang Zihao,Lan Qiuping,Wang Ermin \\ Department of Geomatics, School of Earth Science \& Engineering, HoHai University, Nanjing, \\ 211100, China
}

Keywords: 3-d laser scanning point clouds; curving tunnel; central axis; section cutting; Delaunay triangulation;

\begin{abstract}
For 3-d laser scanning point clouds of an expressway tunnel, a method of automatically extracting complete contours of cross-sections from them is proposed. By taking advantage of abundant normal vectors embedded in point clouds, this method can accurately compute the central axis of the tunnel and build a reference cylinder surface coaxial with the tunnel. To rebuild the surface model for tunnels, a cylindrical projection is used to map all point clouds into a 2-d space firstly, and an improved 2-d Delaunay algorithm which can deal with the border-less circumferential angles is subsequently employed to construct the triangulated mesh from projection points in this two-dimensional space. Following the one-to-one mapping from the original point cloud to the projection point set, a corresponding 3-d triangulated mesh of the original point cloud is finally constructed in accordance with the 2-d triangulation result. By intersecting 3-d triangular mesh of tunnel with cross sections perpendicular to the tangential direction of axis, a series of contours are generated at the specified locations. For the curved tunnel, this method can adjust the direction of sections finely to make them always suitable for the constant change of tunnel direction. At the end of this paper, the experimental result demonstrate the effectiveness and high efficiency of this method by the use of the point clouds acquired from a real highway tunnel.
\end{abstract}

\section{Introduction}

With the total mileage of highways growing to 4.24 million kilometers, by 2012, the total amount of China's highway tunnels has exceeded 10 thousands, and their accumulated length been up to 8.05 million meters. The tunnel is the important engineering facilities, much like the throat of high-speed way, and its' structural health and engineering safety is one important influential factor for convenience and efficiency of the highway network. So the disease detection for tunnels has gradually taken priority over all other road maintenance for the highway owner. Three-dimensional laser scanning, as a new automatic measuring technique, can quickly collect precise geometric information of complete target surface from a distance, and thus can facilitate the measurement of geometric parameters for tunnels without need to shut down the road to traffic. However, from voluminous point clouds acquired from long tunnels at high resolution, how to efficiently retrieve geometric features, including central axis and cross-sectional contours, has become an urgent issue that must be solved at present. Tuo ${ }^{[1]}$ proposes a way to obtain cross sections for tunnel by means of surface fitting to local neighborhood point set. In addition to original scanning points, this method also needs to be provided additional designed parameters about tunnel axis as well, but there is no evidence to suggest that it can ensure accuracy for long-distance tunnels. Xiao ${ }^{[2]}$ takes advantage of a fitting axis to determine a series of perpendicular cross sections which are intersected with tunnel to obtain sectional contours. But there are some limitations on the application of this method to other shapes of tunnels than circle. $\mathrm{Hu}{ }^{[3]}$ develops a way to automatically draw cross-sectional contour lines based on design parameters of tunnel, which definitively does not apply to the reverse engineering of already built tunnels.

In three-dimensional space, cross-sectional contour is the intersection line of inner surface and the cross-section perpendicular to axis. Therefore, for massive point clouds acquired from the real tunnel, as long as the precise central axis is determined and the complete inner surface model constructed, the sectional contour anywhere within the tunnel can be calculated directly by 
intersecting operation of 3d spatial objects.

Following this basic idea, we design an automatic method of continuously outlining cross-sectional contours on triangulated mesh constructed by tunnel point could. The method does not require the tunnel central line given a priori, instead can figure out the direction of the axis based on distribution of point clouds. According to the geometric feature of tunnel, we specially design a cylndrical projection schema to transform the 3-d networking problem into a 2-d space. In order to rebuild tunnel inner surface from 3-d point clouds, we directly take scanning points as vertices to construct the triangulated mesh, which is more consistent with real inner wall of tunnel than smooth surface by fitting the points. At the final experiment, we apply this method to the real laser scanning data acquired from a section of highway tunnel, and the experimental results demonstrate that this method can automatically follow the trend of tunnel to adjust the direction of cross-section and generate closed cross-sectional contours one by one.

\section{Detecting axis direction}

Principle of axis detection. Normal vector is an important geometric attribute of point clouds, and the shifting pattern of normal vectors in bulk can always clearly indicate the geometric shape of target surface. For the scanning point clouds of tunnel, almost every normal vector on the inner surface, including arch crown, dividing wall and roadway, is perpendicular to the axis of tunnel. Therefore, given two nonparallel normal vectors of tunnel points at random, $\overrightarrow{n_{1}}, \overrightarrow{n_{2}}$, which would not even be coplanar, the direction of axis can theoretically be worked out by calculating the cross product of them. However, because there exist unavoidable measurement errors in collecting original points and calculation errors in fitting the surface model to estimate normal vectors, it is absolutely unreliable to determine the final direction of axis based simply on one pair of normal vectors. As shown in Figure 1a and 1b, massive pairs of nearly perpendicular normal vectors are uniformly and symmetrically distributed on the tunnel surface, which should be used as far as possible to reduce effect of these errors on estimates of the axial direction. For a given small section of tunnel point cloud with normal vectors, you can find a certain number of perpendicular normal pairs. By computing cross product, one axial direction can be derived from one pair, so a bunch of approximate directions around truth value would be calculated. With just averaging over those, the random errors hiding in every possible direction can cancel each other out, and the result direction will be closer to the actual value.
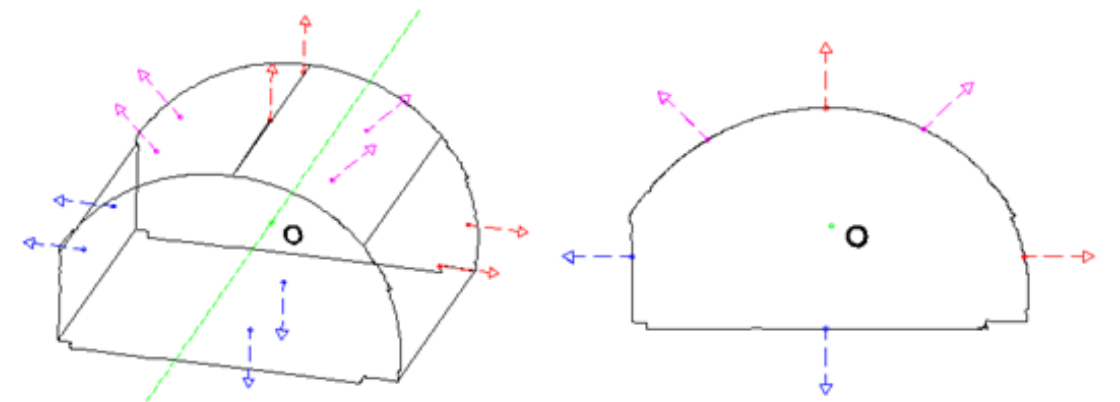

(a) 3D-view

(b) sectional view

Fig.1: Relationship between normal vectors and axial direction

Fitting computation of normal vector.For a given point cloud without normal vectors, computation of the surface normal at every point is the first job of the above-mentioned method. Considering that a 3d laser scanning point cloud is essentially the dense and continuous sampling data set of geometric shape of target surface, data processor often rely on several most neighboring points to approximate the tangent plane to the local surface they are on at the center point. For a given point p, we search the $\mathrm{k}$ nearest neighboring points from the point cloud by $\mathrm{K}-\mathrm{NN}^{[4]}$, and fit a plane on these points centering on point p. So the normal direction of the fitting plane is considered as the direction of normal vector of point $\mathrm{p}$. We compute normal vectors for point clouds of a 5.6-meter tunnel, and the 
result is shown in figure 2 .

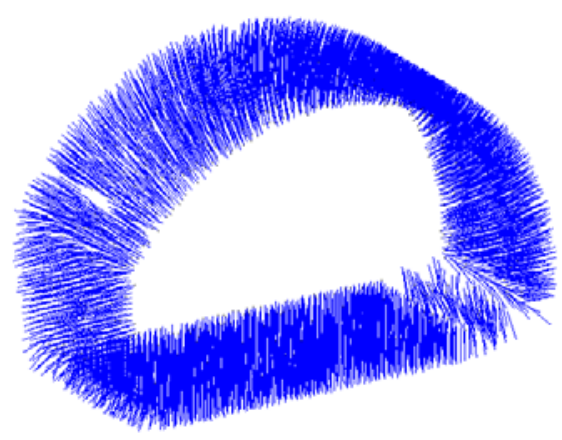

Fig.2: Normal vectors of tunnel

Extraction of axis.For given point clouds of tunnel $\left\{\left(x_{i}, y_{i}, z_{i}\right) \mid i=1,2, \cdots, n\right\}$, the coordinate of central point $O\left(x_{0}, y_{0}, z_{0}\right)$ can be simply calculated by $\left(x_{0}, y_{0}, z_{0}\right)=\sum_{i=1}^{n}\left(x_{i}, y_{i}, z_{i}\right) / n$. Based on normal vectors, the direction of average axis $\vec{f}$ can be work out through the process expressed as follows. For given two nonparallel normal vectors of scanning points at random, $\left(n x_{i}, n y_{i}, n z_{i}\right)$ and $\left(n x_{j}, n y_{j}, n z_{j}\right), i, j \in[1, n]$. If the intersection angle of them appreciates $90^{\circ}$ (formula (1)), $\vec{f}$ can be obtained by formula (2).

$$
\begin{gathered}
\cos ^{-1}\left[\left(n x_{i}, n y_{i}, n z_{i}\right) \bullet\left(n x_{j}, n y_{j}, n z_{j}\right)\right] \in(90-\varepsilon, 90+\varepsilon) \\
\vec{f}=(a, b, c)=\left[\sum\left(n x_{i}, n y_{i}, n z_{i}\right) \otimes\left(n x_{j}, n y_{j}, n z_{j}\right)\right] / k
\end{gathered}
$$

$\otimes$ means the cross product of vectors; $\mathrm{k}$ is the number of pairs of points meeting formula(1); $\varepsilon$ is a minimal angle threshold.

From the above equation (1), (2), it is known that $\mathrm{k}$ depends on threshold $\varepsilon$. Thus, the precision of $\vec{f}$ is strongly linked to threshold $\varepsilon$. Considering that axial direction does direction impact on the final direction, it is essential of the following experiment to access the deviation between $\vec{f}$ and real axis.

Defining the cross product of every pair of normal vectors as a sample, the total number $\mathrm{k}$ of point set is determined by threshold $\varepsilon$, and $\vec{f}$ is the expected value of this sample. According to the regulation of statistical samples, the distributions around the expected value objectively reflect the authenticity of the expected value. Specifically, it also means, more samples deviate from $\vec{f}$ slightly, the axis $\vec{f}$ will be considered in higher reliability.

We define the angle between every sample and the expected value $\vec{f}$ as $\theta$, so as to quantitatively describe the degrees that every sample deviates from $\vec{f}$. On condition of different intervals of $\theta$, counting the corresponding number of samples, which can be used to analysis the effects on $\theta$ caused by different $\varepsilon$.

Given this, the number of samples is defined as $\mathrm{m}$. As an example, on the condition that $\varepsilon=10^{\circ}$ and $\mathrm{k}=353$, the distribution of $\mathrm{m}$ can be described as plot (1) in figure 3 . Obviously, the plot almost fits the normal distribution, yet $\mathrm{m}$ distributes discretely. There exist quite a number of samples distribute around the intervals like $\left(4^{\circ}, 6^{\circ}\right],\left(6^{\circ},+\infty\right)$, which indicates the reliability of $\vec{f}$ comparatively poor.

In order to screen out the closest $\vec{f}$ to the real axis, apply in this tunnel experiment, the threshold $\varepsilon$ is set as different degrees $7^{\circ}, 5^{\circ}, 3^{\circ}$ and $1^{\circ}$, and the distribution of $\theta$ can be described from plot (2) to (5) in figure 3. As threshold $\varepsilon$ lessens, $\mathrm{k}$ is gradually smaller. However, the ratio that number of samples on condition of $\theta \in\left(0^{\circ}, 2^{\circ}\right]$ in every point set is getting higher and higher. 
Therefore, it is believed that $\vec{f}$ in plot(5) is in highest reliability. And, $\vec{f}$ on condition of $\varepsilon=1^{\circ}$ can be adopted in this experiment, the result of which is shown in figure 4 .

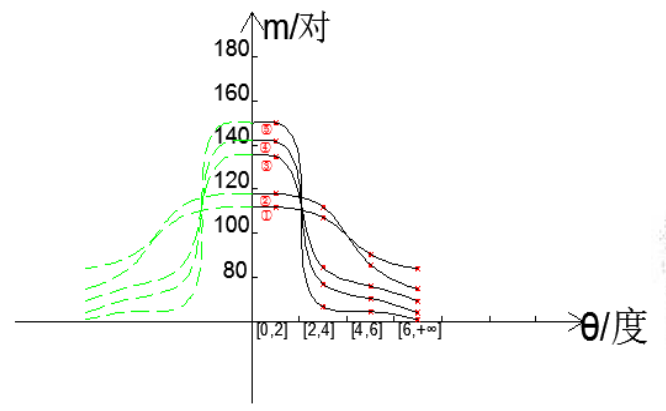

Fig.3: Distribution of $\theta$

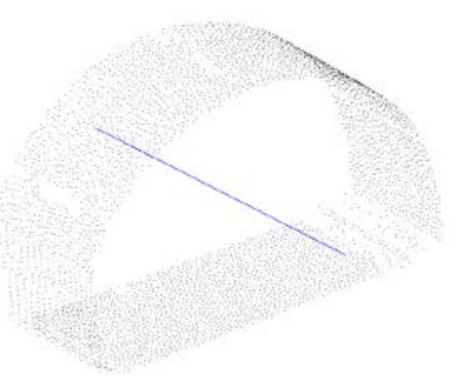

fig.4: Direction of $\vec{f}\left(\varepsilon=1^{\circ}\right)$

D triangulation of internal surface of tunnel.Because point clouds of tunnel are discrete, only build 3D continuous model of surface, can we obtain complete and closed contours of cross-sections based on the intersection of sections and surface model. According to geometric character of tunnel, a method of triangulation of internal surface based on cylindrical projection is designed, which can be expressed as follows. First, on the basis of computed axis, a 2D cylindrical projection coordinate system can be built. After all 3D scanning points are projected to this coordinate system, every point has a 2D projected coordinate. Afterwards, it is fundamental to construct delaunay triangulation network in 2D space, and as well topological relations of that will be mapped back into 3D space. Finally, it is necessary to sew and mend some local areas of the triangulation network to ensure network surface continuous and complete.

\section{Cylindrical projection based on axis}

A projected cylinder coaxial with tunnel, which is perpendicular to axis, can be built in figure 5. For any point p of tunnel, the ray, perpendicular to $\vec{f}$ and crossing point $\mathrm{p}$, is only determined. And the pedal is named p'. By this way of projection, every scanning point of point clouds can be project to only one 2D point $(\alpha, \beta)$ on the cylindrical surface. Certainly, any 2D point $(\alpha, \beta)$ can be uniquely determined by the position of scanning points on the cylindrical surface. For any $2 \mathrm{D}$ point $(\alpha, \beta), \alpha$ refers to the distance from center point $\mathrm{O}$ to the plane of cross-section that is crossing the point $\mathrm{p}$ and perpendicular to $\beta$. In the plane of cross-section, the center point $\mathrm{O}$ is first defined as origin, and as well straight up direction is defined as zero direction. Thus, the angle, which rotates from zero direction to direction of ray crossing the point $\mathrm{p}$, is defined as $\beta$. After $\alpha$ and $\beta$ are defined, $(\alpha, \beta)$ of every scanning point can be figure out according to geometrical characters. Whether to calculate $\alpha$ or $\beta$, it is fundamental to work out the equation of any cross-section and axis $\vec{f}$. The equation of cross-section can be determined according to the axis $\vec{f}(a, b, c)$ and the point $p\left(x_{p}, y_{p}, z_{p}\right)$ as follows.

$$
a\left(x-x_{p}\right)+b\left(y-y_{p}\right)+c\left(z-z_{p}\right)=0
$$

The equation of axis crossing the center point $\mathrm{O}\left(x_{0}, y_{O}, z_{O}\right)$ can be expressed as follows.

$$
\frac{x-x_{0}}{a}=\frac{y-y_{0}}{b}=\frac{z-z_{0}}{c}
$$

Based on simultaneous equation (3), (4), it is easy to get the coordinate of foot point $p^{\prime}\left(x^{\prime}, y^{\prime}, z^{\prime}\right)$. Accordingly, the vertical distance from center point $\mathrm{O}$ to cross-section is $|\alpha|=\sqrt{\left(x_{\mathrm{p}}-x^{\prime}\right)^{2}+\left(y_{p}-y^{\prime}\right)^{2}+\left(z_{p}-z^{\prime}\right)^{2}}$, and the plus-minus sign of $\alpha$ can be determined by the 
relative position of point $\mathrm{p}$ and $\mathrm{O}$. As $\beta$ is defined based on clockwise rotation, when $y_{p}>y_{O}, \beta=\cos ^{-1} \frac{\left|\overrightarrow{p^{\prime} p} \bullet \vec{\beta}_{0}\right|}{\left|\overrightarrow{p^{\prime} p}\right| \bullet\left|\vec{\beta}_{0}\right|}$; when $y_{p}<y_{O}, \beta=2 \pi-\cos ^{-1} \frac{\left|\overrightarrow{p^{\prime} p} \bullet \vec{\beta}_{0}\right|}{\left|\overrightarrow{p^{\prime} p}\right| \bullet\left|\vec{\beta}_{0}\right|}$. And the vertical direction $\vec{\beta}_{0}$ is a unit vector of zero direction.

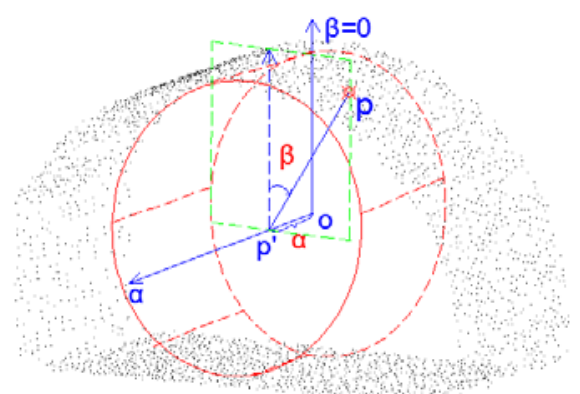

Fig.5: Cylindrical projection system

Delaunay triangulation.After obtaining 2D projected coordinate $(\alpha, \beta)$ of every scanning point, it is critical to 2D delaunay triangulate based on the algorithm proposed by D.T.Lee ${ }^{[5]}$. Considering the data is so vast, it is necessary to optimize efficiency of triangulation by the method raised by Huang ${ }^{[6]}$. According to one-to-one correspondence between scanning point and 2D projected point, the connection of edges in 3D space can be reconstructed, which is in keeping with those in 2D space. Here is the result of reconstruction in 3D space.

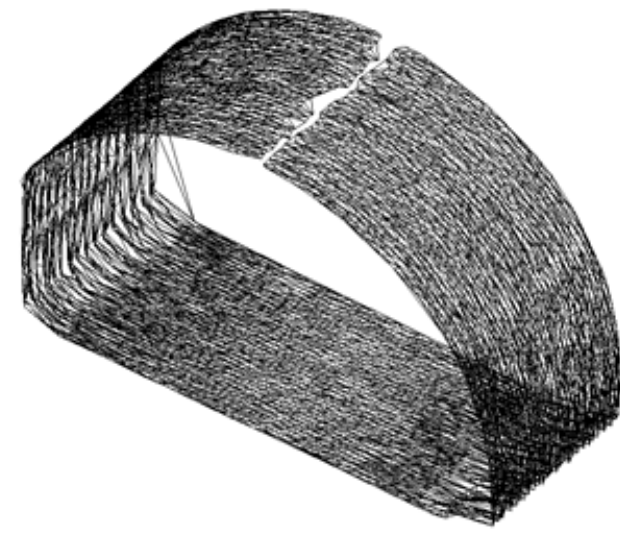

(a) 3D triangulation network

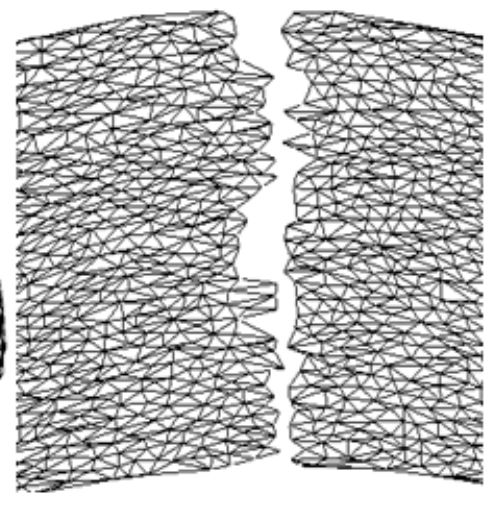

(b) enlargement of crack

Fig.6: Preliminary result of 3D triangulation

Local mending of 3D triangulation network.As figure $6 \mathrm{a}$ and $6 \mathrm{~b}$ show, there exists an obvious crack around the center of tunnel vault, which arises from aforementioned algorithm of projection. It is urgent and important to analyze its specific cause and repair it. Under the environment of monotonic space with boundary, $\beta$ coordinates of all $2 \mathrm{D}$ projected points distribute in $\left[0^{\circ}, 360^{\circ}\right)$. As the boundary values, $0^{\circ}$ is far from $360^{\circ}$. However, in 3D space, the surface of tunnel is clockwise continuous, which means $0^{\circ}$ and $360^{\circ}$ are connected head to tail. Therefore, the algorithm of projection makes this existed connection of edges lost. We call them 0-360 missing edges. 0-360 missing edges consist of two points groups. One group distributes on left of $0^{\circ}$, and the other one distribute on right of $360^{\circ}$. According to this, the crack is generated during the projection from 2D triangulation to $3 \mathrm{D}$ triangulation. Therefore, it is deduced that all 0-360 missing edges are crossing the zero direction of $\beta\left(0^{\circ}\right.$ or $\left.360^{\circ}\right)$.

In order to find back 0-360 missing edges, a solution is designed following. As figure 7a shows, another cylindrical projection system is built for the second time. The zero direction of $\beta$ is rotated $90^{\circ}$ clockwise, which is called $\beta^{\prime}$. By the above-mentioned methods of triangulation and projection, a new 3D triangulation network of tunnel can be obtained. In figure $7 \mathrm{~b}$, it is obvious that a crack lies around the new zero direction of $\beta^{\prime}$. It is also means that the position of crack has been rotated $90^{\circ}$ 
clockwise compared to that of first crack. However, because it is only that the zero direction of $\beta$ is changed during the second triangulation, the position and serial number of original points keep unaltered. And, 0-360 missing edges keep complete here. Therefore, in the same position of tunnel, during the second triangulation can be used to fill and mend the first crack. Finally, the result of repairing edges is shown in figure 8(a) and (b).

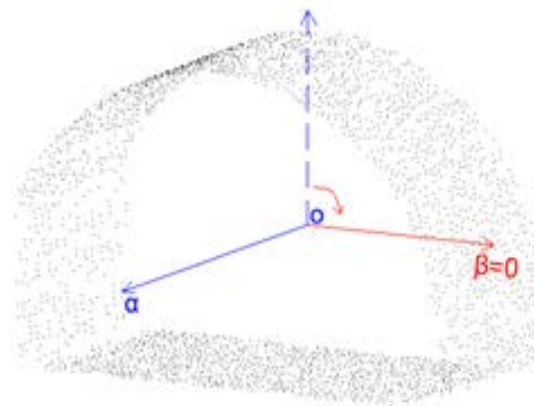

(a)

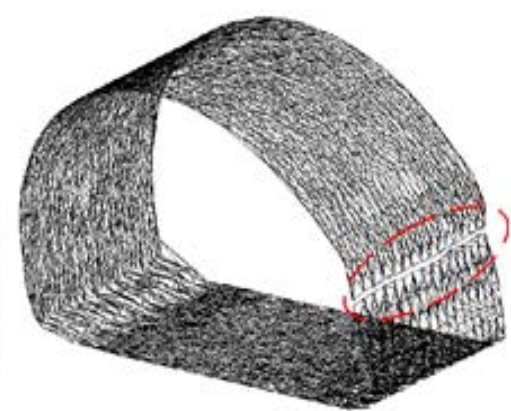

(b)

Fig.7: New $\alpha-\beta$ coordinate system

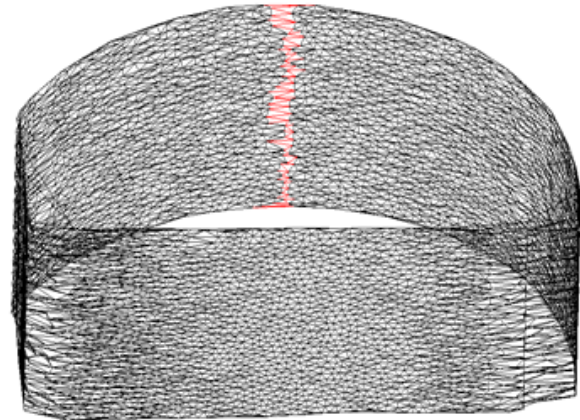

(a)

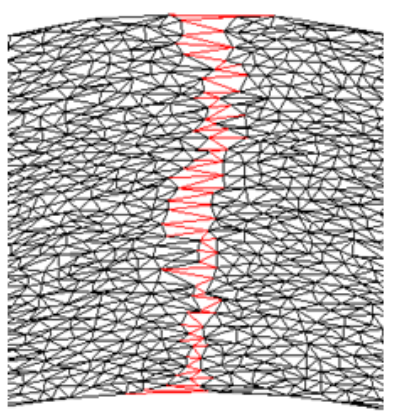

(b)

Fig.8: Crack mending and sewing

\section{Cutting out cross-sections continuously}

As is shown in figure 9, contour of cross-section consists of points intersected by cross-section (red) and edges of triangulation network (blue). The coordinates of point, intersected by any edge $p_{1} p_{2}$ of triangulation network and selected cross-section, can be obtained by combining the equation of cross-section and $p_{1} p_{2}$. Set $p_{1}$ as $\left(x_{1}, y_{1}, z_{1}\right), p_{2}$ as $\left(x_{2}, y_{2}, z_{2}\right)$, and the equation of $p_{1} p_{2}$ can be expressed as follows.

$$
\frac{x-x_{1}}{x_{2}-x_{1}}=\frac{y-y_{1}}{y_{2}-y_{1}}=\frac{z-z_{1}}{z_{2}-z_{1}}
$$

Combining equation (3),(5), and the point $p\left(x_{p}, y_{p}, z_{p}\right)$ can be work out. The point $p$, intersection point of line $p_{1} p_{2}$ and cross-section, is on the segment $p_{1} p_{2}$ when it meets the following conditions.

$\min \left(x_{p_{1}}, x_{p_{2}}\right)<x_{p}<\max \left(x_{p_{1}}, x_{p_{2}}\right) \cap \min \left(y_{p_{1}}, y_{p_{2}}\right)<y_{p}<\max \left(y_{p_{1}}, y_{p_{2}}\right) \cap \min \left(z_{p_{1}}, z_{p_{2}}\right)<z_{p}<\max \left(z_{p_{1}}, z_{p_{2}}\right)$ By this way, the point set of all closed contours can be obtained. In order to get closed and non-overlapping poly line of contour, all disordered points should be sorted on clockwise direction in the plane of section and then connect the points in turn to form the closed contour in figure 10. 

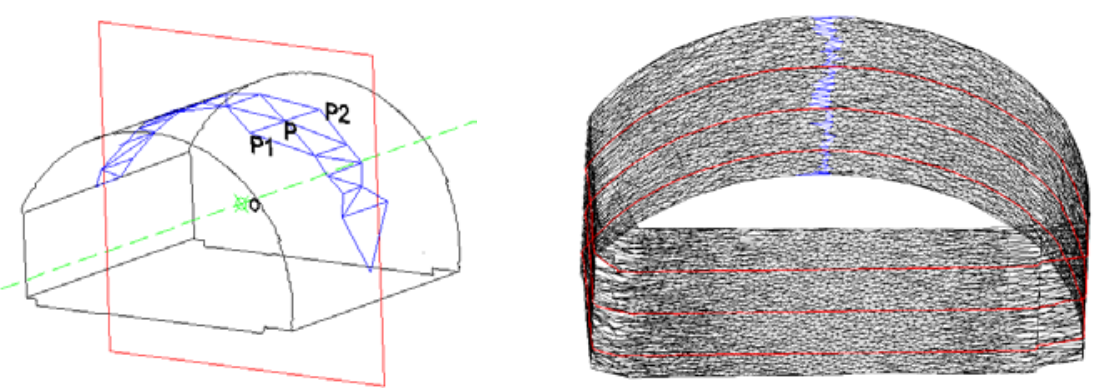

Fig.9:Intersection of cross-section and network fig.10:Contours of cross-sections

\section{Specificity of curving tunnel}

To some extent, most of middle-distance or long highway tunnels are curving. In figure 11(a) and (b), as the trend of tunnel changes, the original axis cannot represent the normal vectors of cross-sections in different positions of tunnel. Although original axis $\vec{f}$ cannot express the trend of tunnel precisely, local fragments of point clouds retain the imperceptible details of trend changing.

According to this character, based on local fragments of point clouds, we design a method of adjusting the position of cross-section precisely. Concrete steps are following. First, viewing point clouds as a whole, the whole trend of tunnel can be determined by computing the direction of original axis. Also, according to original axis, original positions and directions of all cross-sections can be determined roughly. Afterwards, centering on every cross-section, every fragment, much like a local cuboid which is $d$ millimeters in width, are cut out. And based on normal vectors of this fragment, a new and local axis $\vec{f}^{\prime}$, more particularly representing the normal vector of cross-section, come out in need. In the end, contours of cross-sections can be gained by intersecting the new cross-section and triangulation network. And significant improvement of precision is shown in figure 11(b).

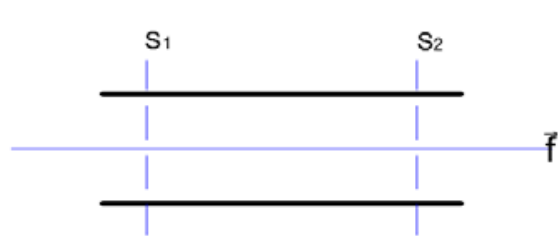

(a) linear tunnel

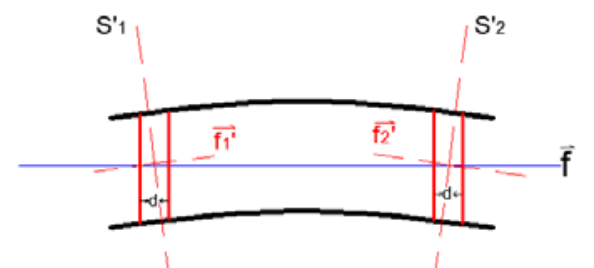

(b) curving tunnel

Fig.11: $\vec{f}$ in different curving degrees of tunnels

\section{Experiment and analysis}

By 3D laser scanner Trimble GX200, we collect the data of TiZishan tunnel in Ninghang highway around boundary of Jiangsu and Zhejiang. The data, including 1804087 scanning points, is a part of tunnel from $0 \mathrm{~m}$ to $130 \mathrm{~m}$. According to engineering design data, there exists an angle about $4.8^{\circ}$ in entrance and exit.

A personal computer, $2.5 \mathrm{GHz} \mathrm{CPU}$ and $4 \mathrm{~GB}$ internal memory, is used to run the program. During the experiment, the threshold $\varepsilon$ is set as $1^{\circ}, 8713$ pairs of samples are adopted. On this occasion, the computed original axis $\vec{f}$ is $(-0.241,-0.133,0.023) \mathrm{m}$. During the period of 3D triangulation, 1634102 triangles are built, including 7182 repaired triangles. During the following step of cutting out contours, local fragment of point clouds in width of 1000 millimeter is put to use. Every other 2 meter starting from the entrance of tunnel, one contour of cross-section is extracted. And in the end, based on the first and last normal vector, the angle of this curving tunnel can be measured as $4.762^{\circ}$, which agrees with design parameters. The result of experiment is shown in figure 13.

As far as program running time is concerned, the experiment spends $682.447 \mathrm{~s}$ computing 63 complete contours of cross-sections. Most of time cost in 2D delaunay triangulation, which takes up 627.247s. For the remaining part, because hash cluster index is built beforehand, it only takes 3.255s 
on computing normal vectors and axis.

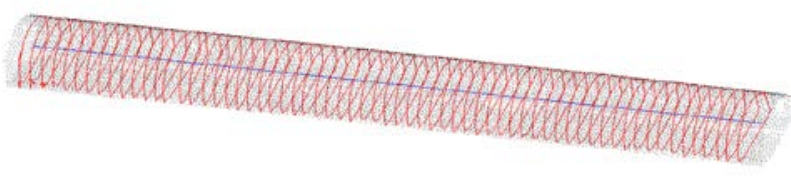

Fig.12: Continuous contours of curving tunnel

\section{Conclusion}

As an important geometrical attribute, normal vector is usually implicit information of point clouds. For local surface of scanning target, normal vector plays a key role of representing its geometrical morphology. Based on information of normal vectors, a method of automatically tracking tunnel trend and precisely adjusting direction of cross-section is designed in this paper. According to geometrical features of tunnel, on the basis of 2D delaunay algorithm, a means to construct 3D triangulation network using cylindrical projection is proposed and tested. For any contour of cross-section in any position of curving or linear tunnel, it can be precisely obtained by intersecting local cross-sections and triangulation network on surface. The method we proposed not only brings the subsidiarity of computer into full play, greatly reduces the manual workload, but also ensures the process of cutting out cross-section in high precision. More valuable, this method can deal with point clouds of tunnel in different cavities and arbitrary degrees of curve, which means it will be extensively applied.

\section{Acknowledgements}

The research work was supported by National Natural Science Foundation of China under Grant No . 41201439 and 41301406, Natural Science Foundation of Jiangsu Province under Grant No. BK20 130829.

\section{References}

[1] TUO Lei1 KANG Zhizhong1 XIE Yuancheng2.Continuously Vertical section Abstraction for Deformation Monitoring of Subway Tunnel Based on Terrestrial Point clouds [J].Geomatics and Information Science of Wuhan University, 2013, 38(2): 171-175.

[2] Xiao Qinghua, Zhang Jichun. Mathematical model of automatically drawing sections of tunnel[J]. Railway Engineering, 2006, 2006(9):29-31.

[3] HU Qijia. Scanning Technology in the Tunnel Deformation Monitoring [D]. Chengdu graduate school of southwest jiao tong university, 2013:1-60.

[4] Lin Xiang,Jiang Suyong,Chen Boliang etc. High-precision Algorithm \& Programming Based on the Plane Fitting of Minimum Condition [J].Journal of Shantou University(Natural Science).2013,28(1):34-39.

[5] Lee.D. T.\&B. J. Schachter. Two algorithms for constructing a Delaunay triangulation[J]. International Journal of Computer \& Information Sciences, 1980, 9(3):219-242.

[6] HUANG Dilong.A Research of Ameliorating Arithmetic about delaunay triangulation [J].Computing techniques for geophysical and Geo-chemical exploration, 2006, 28(1):66-71. 